\title{
New convex functions in linear spaces and Jensen's discrete inequality
}

\section{Xiusu Chen ${ }^{*}$}

\section{"Correspondence:}

xousuchen10@sina.com

School of Mathematics and

Statistics, Chongqing Key laboratory

of Electronic Commerce \& Supply

Chain System, Chongqing

Technology and Business University,

Chongqing, 400067, P.R. China

\begin{abstract}
In the present paper we introduce a new class of s-convex functions defined on a convex subset of a real linear space, establish some inequalities of Jensen's type for this class of functions. Our results in special cases yield some of the recent results on classic convex functions.
\end{abstract}

Keywords: $s$-Orlicz convex functions; s-convex functions; Jensen's inequalities

\section{Introduction}

The research on convexity and generalized convexity is one of the important subjects in mathematical programming, numerous generalizations of convex functions have been proved useful for developing suitable optimization problems (see [1-3]). s-convex functions defined on a space of real numbers was introduced by Orlicz in [4] and was used in the theory of Orlicz spaces. $s$-Orlicz convex sets and $s$-Orlicz convex mappings in linear spaces were introduced by Dragomir in [5]. Some properties of inequalities of Jensen's type for this class of mappings were discussed.

Definition 1.1 [5] Let $X$ be a linear space and $s \in(0, \infty)$. The set $K \subset X$ is called $s$-Orlicz convex in $X$ if the following condition is true:

$$
x, y \in X \text { and } \alpha, \beta \geq 0 \text { with } \alpha^{s}+\beta^{s}=1 \text { imply } \quad \alpha x+\beta y \in X .
$$

Remark If $s=1$, then, by the above definition, we recapture the concept of convex sets in linear spaces.

Definition 1.2 [5] Let $X$ be a linear space and $s \in(0, \infty)$. Let $K \subset X$ be an $s$-Orlicz convex set. The mapping $f: K \rightarrow R$ is called $s$-Orlicz convex on $K$ if for all $x, y \in K$ and $\alpha, \beta \geq 0$ with $\alpha^{s}+\beta^{s}=1$, one has the inequality

$$
f(\alpha x+\beta y) \leq \alpha^{s} f(x)+\beta^{s} f(y)
$$

Note that for $s=1$ we recapture the class of convex functions.

In this paper we introduce another class of $s$-convex functions defined on convex sets in a linear space. Some discrete inequalities of Jensen's type are also obtained.

O2013 Chen; licensee Springer. This is an Open Access article distributed under the terms of the Creative Commons Attribution License (http://creativecommons.org/licenses/by/2.0), which permits unrestricted use, distribution, and reproduction in any medium, provided the original work is properly cited. 


\section{The relations among s-convex functions, s-Orlicz convex functions and convex functions in linear spaces}

Let $X$ be a linear space and $s \in(0, \infty)$ be a fixed positive number, let $K \subset X$ be a convex subset. It is natural to consider the following class of functions.

Definition 2.1 The mapping $f: K \rightarrow R$ is called $s$-convex on $K$ if

$$
f(\alpha x+\beta y) \leq \alpha^{s} f(x)+\beta^{s} f(y)
$$

for all $x, y \in K$ and $\alpha, \beta \geq 0$ with $\alpha+\beta=1$.

Remark 2.2 If $s=1$, then, by the above definition, we recapture the concept of convex functions in linear spaces.

Remark 2.3 s-convex functions defined on a convex set in linear spaces are different from convex functions.

(1) There exist $s$-convex mappings in linear spaces which are not convex for some $s \in(0, \infty) \mid\{1\}$ (see the following Example 1).

(2) When $0<s \leq 1$, every non-negative convex function defined on a convex set in a linear space is also an $s$-convex function. When $s \geq 1$, every non-positive convex function defined on a convex set in a linear space is also an $s$-convex function.

Example 1 Let $\mathrm{X}$ be a normed linear space, let $K=X$ and $0<s \leq 1$, define $f(x)=\|x\|^{s}$ for all $x \in K$. For every $x, y \in K$ and $\alpha, \beta \geq 0$ with $\alpha+\beta=1$, when $\|\alpha x\|=0$, either $\alpha=0$ or $x=0$, therefore, $\alpha x=0$, and

$$
f(\alpha x+\beta y)=f(\beta y)=\|\beta y\|^{s}=\beta^{s}\|y\|^{s}=\beta^{s} f(y) ;
$$

when $\|\alpha x\| \neq 0$, from the monotonous increasing of the function $g(t)=1+t^{s}-(1+t)^{s}$ in the interval $[0, \infty)$, we have $g(t)=1+t^{s}-(1+t)^{s} \geq g(0)=0, \forall t>0$, then

$$
\begin{aligned}
f(\alpha x+\beta y) & =\|\alpha x+\beta y\|^{s} \leq(\alpha\|x\|+\beta\|y\|)^{s}=\alpha^{s}\|x\|^{s}\left[1+\frac{\beta\|y\|}{\alpha\|x\|}\right]^{s} \\
& \leq \alpha^{s}\|x\|^{s}\left[1+\left(\frac{\beta\|y\|}{\alpha\|x\|}\right)^{s}\right]=\alpha^{s}\|x\|^{s}+\beta^{s}\|y\|^{s}=\alpha^{s} f(x)+\beta^{s} f(y),
\end{aligned}
$$

hence $f$ is an $s$-convex function on $X$; however, $f$ is not a convex function on $X$ with $0<$ $s<1$.

Remark 2.4 There exist $s$-Orlicz convex functions in linear spaces which are not $s$-convex functions for some $s \in(0, \infty) \mid\{1\}$.

Example $20<s \leq 1, K=R^{2}$. Consider the set $K=\left\{(x, y) \in R^{2}:|x|^{s}+|y|^{s} \leq 1\right\} \subset R^{2}$. Let $X_{1}=\left(x_{1}, y_{1}\right), X_{2}=\left(x_{2}, y_{2}\right) \in K$, and let $\alpha, \beta \geq 0$ with $\alpha^{s}+\beta^{s}=1$. Then

$$
\left|x_{1}\right|^{s}+\left|y_{1}\right|^{s} \leq 1, \quad\left|x_{2}\right|^{s}+\left|y_{2}\right|^{s} \leq 1,
$$


which imply

$$
\begin{aligned}
\left|\alpha x_{1}+\beta x_{2}\right|^{s} & \leq\left(\alpha\left|x_{1}\right|+\beta\left|x_{2}\right|\right)^{s}=\left\{\alpha\left|x_{1}\right|\left(1+\frac{\beta\left|x_{2}\right|}{\alpha\left|x_{1}\right|}\right)\right\}^{s}=\left[\alpha\left|x_{1}\right|\right]^{s}\left(1+\frac{\beta\left|x_{2}\right|}{\alpha\left|x_{1}\right|}\right)^{s} \\
& \leq\left[\alpha\left|x_{1}\right|\right]^{s}\left[1+\left(\frac{\beta\left|x_{2}\right|}{\alpha\left|x_{1}\right|}\right)^{s}\right]=\left[\alpha\left|x_{1}\right|\right]^{s}+\left[\beta\left|x_{2}\right|\right]^{s} \leq \alpha^{s}\left|x_{1}\right|^{s}+\beta^{s}\left|x_{2}\right|^{s}
\end{aligned}
$$

and

$$
\left|\alpha y_{1}+\beta y_{2}\right|^{s} \leq \alpha^{s}\left|y_{1}\right|^{s}+\beta^{s}\left|y_{2}\right|^{s}
$$

As

$$
\begin{aligned}
& \alpha X_{1}+\beta X_{2}=\left(\alpha x_{1}+\beta x_{2}, \alpha y_{1}+\beta y_{2}\right) \quad \text { and } \\
& \begin{aligned}
\left|\alpha x_{1}+\beta x_{2}\right|^{s}+\left|\alpha y_{1}+\beta y_{2}\right|^{s} & \leq \alpha^{s}\left|x_{1}\right|^{s}+\beta^{s}\left|x_{2}\right|^{s}+\alpha^{s}\left|y_{1}\right|^{s}+\beta^{s}\left|y_{2}\right|^{s} \\
& =\alpha^{s}\left(\left|x_{1}\right|^{s}+\left|y_{1}\right|^{s}\right)+\beta^{s}\left(\left|x_{2}\right|^{s}+\left|y_{2}\right|^{s}\right)=\alpha^{s}+\beta^{s}=1,
\end{aligned}
\end{aligned}
$$

we deduce that $\alpha X_{1}+\beta X_{2} \in K$, i.e., $K$ is $s$-Orlicz convex in $R^{2}$.

Define $f(u)=\left(x^{2}+y^{2}\right)^{\frac{s}{2}}, u=(x, y) \in K$, we will show that $f$ is an $s$-Orlicz convex function, $f$ is not an $s$-convex function, for $K$ is not a convex subset when $0<s<1$. Since $u_{0}=(1,0) \in$ $K, v_{0}=(0,1) \in K$, but $\frac{1}{2} u_{0}+\frac{1}{2} v_{0}=\left(\frac{1}{2}, \frac{1}{2}\right) \notin K$ for $\left(\frac{1}{2}\right)^{s}+\left(\frac{1}{2}\right)^{s}=\frac{2}{2^{s}}>1$ with $0<s<1$.

\section{Properties of $s$-convex functions in linear spaces}

We consider the following properties of $s$-convex functions in linear spaces.

Theorem 3.1 Let $K \subset X$ be a convex set, $f_{i}: K \rightarrow R$ be s-convex functions, $i=1,2, \ldots, n$. Then

(1) $h(x)=\max _{1 \leq i \leq n} f_{i}(x)$ is an s-convex function on $K$.

(2) $a f_{1}(x)+b f_{2}(x)$ is an s-convex function on $K$ for all $a, b \geq 0$.

The proof is omitted.

Proposition 3.2 Let $f: K \rightarrow R$ be an s-convex function on $K$ and $\gamma \in R$ so that $K_{\gamma}(f)=$ $\{x \in K: f(x) \leq \gamma\}$ is nonempty. Then $K_{\gamma}(f)$ is a convex subset of $K$ when either one of the following two conditions is satisfied:

(1) $\gamma \geq 0$ and $s \geq 1$,

(2) $\gamma \leq 0$ and $1>s>0$.

Proof Let $x_{1}, x_{2} \in K_{\gamma}(f)$ and $\alpha, \beta \geq 0$ so that $\alpha+\beta=1$. Then $f\left(x_{1}\right) \leq \gamma$ and $f\left(x_{2}\right) \leq \gamma$ which imply that

$$
f\left(\alpha x_{1}+\beta x_{2}\right) \leq \alpha^{s} f\left(x_{1}\right)+\beta^{s} f\left(x_{2}\right) \leq \alpha^{s} \gamma+\beta^{s} \gamma=\left(\alpha^{s}+\beta^{s}\right) \gamma
$$

Then $f\left(\alpha x_{1}+\beta x_{2}\right) \leq\left(\alpha^{s}+\beta^{s}\right) \gamma \leq \gamma$ when either one of (1) and (2) is satisfied, which shows that $K_{\gamma}(f)$ is a convex subset of $K$. 
Theorem 3.3 Let $X$ be a linear space, let $K \subset X$ be a convex set, mapping $f: K \rightarrow R$, the following statements are equivalent:

(1) $f$ is an s-convex function on $K$.

(2) For every $x_{1}, x_{2}, \ldots, x_{n} \in K$ and non-negative real number $\alpha_{1}, \alpha_{2}, \ldots, \alpha_{n}$ with $\sum_{i=1}^{n} \alpha_{i}=1$, we have that

$$
f\left(\sum_{i=1}^{n} \alpha_{i} x_{i}\right) \leq \sum_{i=1}^{n} \alpha_{i}^{s} f\left(x_{i}\right)
$$

Proof $(2) \Rightarrow(1)$. This is obvious.

(1) $\Rightarrow(2)$. We will prove by induction over $n \in N, n \geq 2$. For $n=2$, the inequality is obvious by Definition 2.1. Suppose that the above inequality is valid for all $n-1$. For natural number $n$, let $x_{1}, x_{2}, \ldots, x_{n} \in K$ and $\alpha_{1}, \alpha_{2}, \ldots, \alpha_{n} \geq 0$ with $\sum_{i=1}^{n} \alpha_{i}=1$.

If there is some $\alpha_{i}=0$, then delete the number $\alpha_{i}$ and for the remaining $n-1$ number, inequality (3.1) is obvious by using the inductive hypothesis.

Now suppose $\alpha_{i} \neq 0, \forall i=1, \ldots, n$, let $\beta_{1}=\sum_{i=1}^{n-1} \alpha_{i}, \beta_{2}=\alpha_{n}, y_{1}=\frac{1}{\beta_{1}} \sum_{i=1}^{n-1} \alpha_{i} x_{i}, y_{2}=x_{n}$, since $\sum_{i=1}^{n-1} \frac{\alpha_{i}}{\beta_{1}}=1$. Using the inductive hypothesis, we have

$$
f\left(y_{1}\right)=f\left(\sum_{i=1}^{n-1} \frac{\alpha_{i}}{\beta_{1}} x_{i}\right) \leq \sum_{i=1}^{n-1}\left(\frac{\alpha_{i}}{\beta_{1}}\right)^{s} f\left(x_{i}\right)=\frac{1}{\beta_{1}^{s}} \sum_{i=1}^{n-1} \alpha_{i}^{s} f\left(x_{i}\right) .
$$

Then

$$
\begin{aligned}
f\left(\sum_{i=1}^{n} \alpha_{i} x_{i}\right) & =f\left(\beta_{1} y_{1}+\beta_{2} y_{2}\right) \leq \beta_{1}^{s} f\left(y_{1}\right)+\beta_{2}^{s} f\left(x_{i}\right) \\
& \leq \sum_{i=1}^{n-1} \alpha_{i}^{s} f\left(x_{i}\right)+\alpha_{n}^{s} f\left(x_{n}\right)=\sum_{i=1}^{n} \alpha_{i}^{s} f\left(x_{i}\right),
\end{aligned}
$$

and the theorem is proved.

The following corollaries are different formulations of the above inequalities of Jensen's type.

Corollary 3.4 Let $f: K \rightarrow R$ be an s-convex function, $\alpha_{1}, \alpha_{2}, \ldots, \alpha_{n}$ be non-negative real numbers, $P_{n}=\sum_{i=1}^{n} \alpha_{i}>0$. For every $x_{1}, x_{2}, \ldots, x_{n} \in K$, we have that

$$
f\left(\frac{1}{P_{n}} \sum_{i=1}^{n} \alpha_{i} x_{i}\right) \leq \frac{1}{P_{n}^{s}} \sum_{i=1}^{n} \alpha_{i}^{s} f\left(x_{i}\right) .
$$

Corollary 3.5 Let $f: K \rightarrow R$ be an s-convex function, $x_{1}, x_{2}, \ldots, x_{n} \in K$, then we have

$$
f\left(\frac{1}{n} \sum_{i=1}^{n} x_{i}\right) \leq \frac{1}{n^{s}} \sum_{i=1}^{n} f\left(x_{i}\right)
$$


Corollary 3.6 Let $f: K \rightarrow R$ be an s-convex function, $x_{1}, x_{2}, \ldots, x_{n} \in K$. For every nonnegative real number $\alpha_{1}, \alpha_{2}, \ldots, \alpha_{n}$, when $Q_{n}=\sum_{i=1}^{n} \alpha_{i}^{\frac{1}{s}}>0$, then we have

$$
f\left(\frac{1}{Q_{n}} \sum_{i=1}^{n} \alpha_{i}^{\frac{1}{s}} x_{i}\right) \leq \frac{1}{Q_{n}^{s}} \sum_{i=1}^{n} \alpha_{i} f\left(x_{i}\right) .
$$

We consider the following function:

$$
\theta(I, P, f, x):=\sum_{i \in I} \alpha_{i}^{s} f\left(x_{i}\right)-\left(\sum_{i \in I} \alpha_{i}\right)^{s} f\left(\frac{1}{\sum_{i \in I} \alpha_{i}} \sum_{i \in I} \alpha_{i} x_{i}\right),
$$

where $\alpha_{i}>0, i \in I, f$ is an s-convex function on the convex set $K, x_{i} \in K, i \in I, I \in P(N)$, where $P(N)$ denotes the finite subsets of the natural number set $N$.

Theorem 3.7 Let $f: K \rightarrow R$ be an s-convex function, $\alpha_{i}>0, x_{i} \in K, i \in N$, then

(1) $\forall I, J \in P(N), I \cap J=\Phi$, we have that

$$
\theta(I \cup J, P, f, x) \geq \theta(I, P, f, x)+\theta(J, P, f, x) \geq 0 ;
$$

(2) $\forall I, J \in P(N), \Phi \neq J \subset I$, we have

$$
\theta(I, P, f, x) \geq \theta(J, P, f, x) \geq 0,
$$

that is, the mapping $\theta(I, P, f, x)$ is monotonic non-decreasing in the first variable on $P(N)$.

Proof (1) Let $\forall I, J \in P(N), I \cap J=\Phi$. Then we have

$$
\begin{aligned}
\theta(I \cup J, P, f, x)= & \sum_{i \in I} \alpha_{i}^{s} f\left(x_{i}\right)+\sum_{i \in J} \alpha_{i}^{s} f\left(x_{i}\right)-\left(\sum_{i \in I \cup J} \alpha_{i}\right)^{s} f\left(\frac{\sum_{i \in I} \alpha_{i} x_{i}+\sum_{i \in J} \alpha_{i} x_{i}}{\sum_{i \in I \cup J} \alpha_{i}}\right) \\
= & \sum_{i \in I} \alpha_{i}^{s} f\left(x_{i}\right)+\sum_{i \in J} \alpha_{i}^{s} f\left(x_{i}\right) \\
& -\left(\sum_{i \in I \cup J} \alpha_{i}\right)^{s} f\left(\frac{\sum_{i \in I} \alpha_{i}}{\sum_{i \in I \cup J} \alpha_{i}} \frac{\sum_{i \in I} \alpha_{i} x_{i}}{\sum_{i \in I} \alpha_{i}}+\frac{\sum_{i \in J} \alpha_{i}}{\sum_{i \in I \cup J} \alpha_{i}} \frac{\sum_{i \in J} \alpha_{i} x_{i}}{\sum_{i \in J} \alpha_{i}}\right) \\
\geq & \sum_{i \in I} \alpha_{i}^{s} f\left(x_{i}\right)-\left(\sum_{i \in I} \alpha_{i}\right)^{s} f\left(\frac{\sum_{i \in I} \alpha_{i} x_{i}}{\sum_{i \in I} \alpha_{i}}\right) \\
& +\sum_{i \in J} \alpha_{i}^{s} f\left(x_{i}\right)-\left(\sum_{i \in J} \alpha_{i}\right)^{s} f\left(\frac{\sum_{i \in J} \alpha_{i} x_{i}}{\sum_{i \in J} \alpha_{i}}\right) \\
= & \theta(I, P, f, x)+\theta(J, P, f, x)
\end{aligned}
$$

and inequality (3.2) is proved.

(2) Suppose that $I, J \in P(N)$, with $\Phi \neq J \subset I$ and $J \neq I$.

With the above assumptions, consider the sequence

$$
\theta(I, P, f, x)=\theta(J \cup(I \mid J), P, f, x) \geq \theta(J, P, f, x)+\theta(I \mid J, P, f, x)
$$


whence we get

$$
\theta(I, P, f, x)-\theta(J, P, f, x) \geq \theta(I \mid J, P, f, x) \geq 0
$$

and inequality (3.3) is proved.

With the above assumptions, consider the sequence

$$
\theta_{n}:=\sum_{i=1}^{n} \alpha_{i}^{s} f\left(x_{i}\right)-\left(\sum_{i=1}^{n} \alpha_{i}\right)^{s} f\left(\frac{1}{\sum_{i=1}^{n} \alpha_{i}} \sum_{i=1}^{n} \alpha_{i} x_{i}\right), \quad n>2 .
$$

Corollary 3.8 With the above assumptions,

$$
\theta_{n} \geq \theta_{n-1} \geq \cdots \geq \theta_{2} \geq 0
$$

i.e., the sequence $\theta_{n}$ is non-decreasing and one has the inequality

$$
\theta_{n} \geq \max _{1 \leq i \leq j \leq n}\left[\alpha_{i}^{s} f\left(x_{i}\right)+\alpha_{j}^{s} f\left(x_{j}\right)-\left(\alpha_{i}+\alpha_{j}\right)^{s} f\left(\frac{\alpha_{i} x_{i}+\alpha_{j} x_{j}}{\alpha_{i}+\alpha_{j}}\right)\right] \geq 0 .
$$

Theorem 3.9 Let $f: K \subset X \rightarrow R$ be an s-convex function, and $\alpha_{i} \geq 0$ so that $\sum_{i=1}^{n} \alpha_{i}=1$. Let $x_{i j} \in X, 0 \leq i, j \leq n$. Then one has the inequalities

$$
\sum_{i=1}^{n} \alpha_{i}^{s} \alpha_{j}^{s} f\left(x_{i j}\right) \geq \max \{A, B\} \geq \min \{A, B\} \geq f\left[\sum_{i=1}^{n} \alpha_{i} \alpha_{j} x_{i j}\right],
$$

where $A:=\sum_{i=1}^{n} \alpha_{i}^{s} f\left(\sum_{j=1}^{n} \alpha_{j} x_{i j}\right), B:=\sum_{j=1}^{n} \alpha_{j}^{s} f\left(\sum_{i=1}^{n} \alpha_{i} x_{i j}\right)$.

Theorem 3.10 Let $f: K \subset X \rightarrow R$ be an s-convex function, and $\alpha_{i} \geq 0$ so that $\sum_{i=1}^{n} \alpha_{i}=1$. Then, for every $\alpha, \beta \geq 0$, with $\alpha+\beta=1$, we have

(1) $\left(\sum_{j=1}^{n} \alpha_{j}^{s}\right)\left(\alpha^{s}+\beta^{s}\right) \sum_{i=1}^{n} \alpha_{i}^{s} f\left(x_{i}\right) \geq \sum_{i, j=1}^{n} \alpha_{i}^{s} \alpha_{j}^{s} f\left(\alpha x_{i}+\beta x_{j}\right)$,

(2) $\sum_{i, j=1}^{n} \alpha_{i}^{s} \alpha_{j}^{s} f\left(\alpha x_{i}+\beta x_{j}\right) \geq 2^{s-1} \sum_{i, j=1}^{n} \alpha_{i}^{s} \alpha_{j}^{s} f\left(\frac{x_{i}+x_{j}}{2}\right)$,

(3) $\sum_{i, j=1}^{n} \alpha_{i}^{s} \alpha_{j}^{s} f\left(\frac{x_{i}+x_{j}}{2}\right) \geq f\left(\sum_{i=1}^{n} \alpha_{i} x_{i}\right)$,

(4) $\left(\alpha^{s}+\beta^{s}\right) \sum_{i=1}^{n} \alpha_{i} f\left(x_{i}\right) \geq \sum_{i, j=1}^{n} \alpha_{i} \alpha_{j} f\left(\alpha x_{i}+\beta x_{j}\right)$.

Proof By the $s$-convexity of $f$, we can state

$$
\begin{aligned}
& f\left(\alpha x_{i}+\beta x_{j}\right) \leq \alpha^{s} f\left(x_{i}\right)+\beta^{s} f\left(x_{j}\right) \\
& \quad \Rightarrow \quad \sum_{i, j=1}^{n} \alpha_{i}^{s} \alpha_{j}^{s} f\left(\alpha x_{i}+\beta x_{j}\right) \leq \alpha^{s} \sum_{i, j=1}^{n} \alpha_{i}^{s} \alpha_{j}^{s} f\left(x_{i}\right)+\beta^{s} \sum_{i, j=1}^{n} \alpha_{i}^{s} \alpha_{j}^{s} f\left(x_{j}\right)
\end{aligned}
$$




$$
\begin{aligned}
& =\alpha^{s} \sum_{j=1}^{n} \alpha_{j}^{s} \sum_{i=1}^{n} \alpha_{i}^{s} f\left(x_{i}\right)+\beta^{s} \sum_{i=1}^{n} \alpha_{i}^{s} \sum_{j=1}^{n} \alpha_{j}^{s} f\left(x_{j}\right) \\
& =\left(\sum_{j=1}^{n} \alpha_{j}^{s}\right)\left(\alpha^{s}+\beta^{s}\right) \sum_{i=1}^{n} \alpha_{i}^{s} f\left(x_{i}\right) .
\end{aligned}
$$

We get the first inequality (1) in Theorem 3.10.

By the following inequality

$$
f\left(\frac{x_{i}+x_{j}}{2}\right)=f\left(\frac{\alpha x_{i}+\beta x_{j}+\alpha x_{j}+\beta x_{i}}{2}\right) \leq \frac{1}{2^{s}}\left[f\left(\alpha x_{i}+\beta x_{j}\right)+f\left(\alpha x_{j}+\beta x_{i}\right)\right]
$$

we have

$$
\begin{aligned}
\sum_{i, j=1}^{n} \alpha_{i}^{s} \alpha_{j}^{s} f\left(\frac{x_{i}+x_{j}}{2}\right) & \leq \frac{1}{2^{s}}\left[\sum_{i, j=1}^{n} \alpha_{i}^{s} \alpha_{j}^{s} f\left(\alpha x_{i}+\beta x_{j}\right)+\sum_{i, j=1}^{n} \alpha_{i}^{s} \alpha_{j}^{s} f\left(\alpha x_{j}+\beta x_{i}\right)\right] \\
& =\frac{1}{2^{s-1}}\left[\sum_{i, j=1}^{n} \alpha_{i}^{s} \alpha_{j}^{s} f\left(\alpha x_{i}+\beta x_{j}\right)\right] .
\end{aligned}
$$

We get the second inequality (2) in Theorem 3.10.

Let $x_{i j}=\frac{x_{i}+x_{j}}{2}$, by the inequality in Theorem 3.9 , we have

$$
\sum_{i, j=1}^{n} \alpha_{i}^{s} \alpha_{j}^{s} f\left(\frac{x_{i}+x_{j}}{2}\right) \geq f\left(\sum_{i, j=1}^{n} \alpha_{i} \alpha_{j} \frac{x_{i}+x_{j}}{2}\right)=f\left(\sum_{i=1}^{n} \alpha_{i} x_{i}\right),
$$

i.e., inequality (3) is obtained.

$$
\begin{aligned}
\sum_{i, j=1}^{n} \alpha_{i} \alpha_{j} f\left(\alpha x_{i}+\beta x_{j}\right) & \leq \sum_{i, j=1}^{n} \alpha_{i} \alpha_{j} \alpha^{s} f\left(x_{i}\right)+\sum_{i, j=1}^{n} \alpha_{i} \alpha_{j} \beta^{s} f\left(x_{j}\right) \\
& =\left(\alpha^{s}+\beta^{s}\right) \sum_{i=1}^{n} \alpha_{i} f\left(x_{i}\right)
\end{aligned}
$$

i.e., inequality (4) is proved.

\section{Competing interests}

The author declares that they have no competing interests.

\section{Acknowledgements}

The author has greatly benefited from the referee's report. So I wish to express our gratitude to the reviewer and the associate editor SS Dragomir for their valuable suggestions which improved the content and presentation of the paper. The work was supported by the project of Chongqing Municipal Education Commission (No. KJ090732) and special fund project of Chongqing Key laboratory of Electronic Commerce \& Supply Chain System (CTBU).

\section{Received: 20 March 2013 Accepted: 30 August 2013 Published: 07 Nov 2013}

\section{References}

1. Chen, XS: Some properties of semi E-convex functions. J. Math. Anal. Appl. 275, 251-262 (2002)

2. Youness, EA: E-convex sets, E-convex functions and E-convex programming. J. Optim. Theory Appl. 102, 439-450 (1999)

3. Yang, XM: On E-convex sets, E-convex functions and E-convex programming. J. Optim. Theory Appl. 109, 699-703 (2001) 
4. Orlicz, W: A note on modular spaces, I. Bull. Acad. Pol. Sci., Sér. Sci. Math. Astron. Phys. 9, 157-162 (1961)

5. Dragomir, SS: s-Orlicz convex functions in linear spaces and Jensen's discrete inequality. J. Math. Anal. Appl. 210 419-439 (1997)

10.1186/1029-242X-2013-472

Cite this article as: Chen: New convex functions in linear spaces and Jensen's discrete inequality. Journal of Inequalities and Applications 2013, 2013:472

Submit your manuscript to a SpringerOpen ${ }^{\circ}$ journal and benefit from:

- Convenient online submission

- Rigorous peer review

- Immediate publication on acceptance

- Open access: articles freely available online

- High visibility within the field

- Retaining the copyright to your article 